\title{
Ultimate boundedness sufficient conditions for nonlinear systems using TS fuzzy modelling
}

\author{
Michele C. Valentino $^{a}$, Flávio A. Faria ${ }^{\mathrm{b}}$, Vilma A. Oliveira ${ }^{\mathrm{c}, *}$, Luís F.C. Alberto ${ }^{\mathrm{c}}$ \\ a Universidade Tecnológica Federal do Paraná, Campus Cornélio Procópio, Brazil \\ b Universidade Estadual Paulista (Unesp), Instituto de Química, Araraquara, Brazil \\ ${ }^{\mathrm{c}}$ Universidade de São Paulo, Campus São Carlos, Brazil
}

Received 18 August 2016; received in revised form 3 December 2017; accepted 14 March 2018

Available online 20 March 2018

\begin{abstract}
Using Takagi-Sugeno (TS) fuzzy modelling, sufficient conditions to ensure ultimate boundedness of solutions of nonlinear switched systems are given. The sufficient conditions are given in terms of properties of invariant sets and of an auxiliary system formed by a convex combination of the switching subsystems. By exploring the results of this paper, estimates of the attractor and domain of attraction can be found even when (i) the derivative of an auxiliary function V, which plays the same role of a Lyapunov function, attains positive values in some sets and (ii) the solutions of each subsystem of the switched system are not necessarily ultimately bounded. The sufficient conditions are formulated as a problem of checking the feasibility of linear matrix inequalities (LMIs). Indeed, these LMIs provide a systematic procedure that can help to find auxiliary scalar Lyapunov-like functions for a class of switched nonlinear systems. A numerical example illustrates the effectiveness of the proposed approach in estimating attractors of nonlinear dynamic switched systems.
\end{abstract}

(c) 2018 Elsevier B.V. All rights reserved.

Keywords: Invariant sets; Switched systems; TS fuzzy modelling; Ultimate boundedness

\section{Introduction}

Switched nonlinear systems arise in practice when modelling the operation of many engineering systems [1-4]. Although switching is not a new concept in engineering, in the past decade, the theory of switched systems has attracted the attention of many researchers. As a consequence, the stability theory for switched nonlinear systems has significantly developed in this period and in particular the stability theory of a common equilibrium of all subsystems has been addressed by many researchers [5-7]. However, some switched systems do not exhibit a stable equilibrium point, although they are practically stable in the sense that their trajectories enter and stay in a bounded set. As

\footnotetext{
* Corresponding author.

E-mail addresses: valentino@utfpr.edu.br (M.C. Valentino), flaviof15@iq.unesp.br (F.A. Faria), voliveira@usp.br (V.A. Oliveira), lfcalberto@usp.br (L.F.C. Alberto).
} 
a consequence, there has been interest in studying the asymptotic behaviour and, in particular, ultimately bounded properties of solutions.

Lyapunov-like functions are often used to analyse the asymptotic behaviour of system solutions. A key property is the non-positiveness of the derivative of the Lyapunov-like function along solutions. Finding Lyapunov-like functions, satisfying all the assumptions of Lyapunov stability theorems, is usually a difficult task for many nonlinear dynamical systems.

Takagi-Sugeno (TS) fuzzy modelling is useful to help to assess the stability of nonlinear systems and to find Lyapunov-like functions. A large number of approaches for stability analysis and controller synthesis have been developed for TS fuzzy models [1,8-13]. Non-quadratic Lyapunov functions, for example, have been used to study local stability of TS fuzzy systems $[9,14,15]$. However, in these results, the proposed LMIs are established in terms of bounds on the membership functions time derivatives. In [16-18], the proposed LMIs are formulated without the use of bounds on the membership functions time derivatives by exploring properties of membership functions. These results are for asymptotic stability and the derivative of the Lyapunov function along the solution of the nonlinear system is required to be negative.

In the last years the interest to study switched TS fuzzy systems has grown [19-23]. For instance, sufficient conditions for quadratic asymptotic stability and state dependent switching conditions can be found in [19] for a common Lyapunov function, in [20] for a piecewise Lyapunov function and in [21-23] for a fuzzy Lyapunov function.

It is well known that certain switched linear systems with unstable subsystems can be stabilized by a proper switching law [24]. For general nonlinear switched systems, a similar result, where the concept of stability is parallel to the concept of ultimately bounded solutions, is expected.

In this paper, sufficient conditions to ensure the existence of a switching law to guarantee that solutions of a nonlinear system are ultimately bounded are derived even for the case the switched system has subsystems that are not practically stable (no ultimately bounded solutions). These conditions are given in terms of properties of an auxiliary dynamic system, formed by a convex combination of subsystems of the switched system, and in terms of properties of positively invariant sets. The results are first developed for a class of nonlinear switched system and this is accomplished by allowing the value of an auxiliary non-quadratic function $V$, along the solutions of a convex combination of the subsystems, to increase in some sets. In the sequence, the results are applied to switched TS fuzzy systems converting the problem of checking the existence of a switching law, which ensures practical stability, that is, the existence of a bounded attractor for the nonlinear switched system, into a problem of checking properties of some sets and the feasibility of a set of LMIs. The new results are based on the Finsler Lemma [9], which includes a slack matrix variable to reduce the conservativeness of the LMIs conditions and do not impose upper bounds on the time derivative of membership functions which were converted into an equivalent problem of finding positively invariant sets. Estimates of the attractor and stability region (area of attraction) of switched systems described by TS fuzzy systems are obtained, with the results of this paper, in the form of invariant level sets of the auxiliary scalar function.

Throughout this paper, $\bar{\Omega}$ denotes the closure and $\Omega^{c}$ the complement of set $\Omega$, respectively, the notation $\mathbf{P} \succ \mathbf{0}$ $(\mathbf{P} \succeq \mathbf{0})$ indicates that $\mathbf{P}$ is a real symmetric and positive definite (semi-definite) matrix and the notation $\mathbf{P} \prec \mathbf{0}(\mathbf{P} \preceq \mathbf{0})$ indicates that $\mathbf{P}$ is a symmetric and negative definite (semi-definite) matrix, the symbol " $\star$ " within a matrix represents the symmetric terms of the matrix and $e_{i}$ denotes a vector with one at entry $i$ and zeros elsewhere, that is, $e_{i}=$

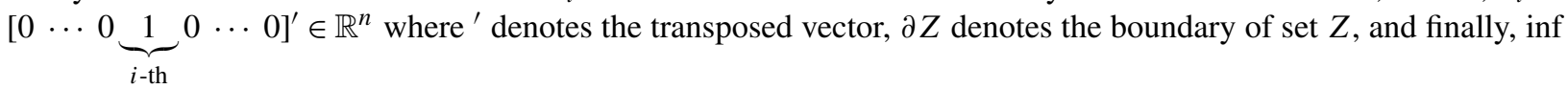
and sup denote the infimum and supremum of a subset, respectively.

\section{Switched systems}

Let us consider the following switched nonlinear system

$$
\dot{x}(t)=f_{\sigma}(x(t))
$$

where $x(t) \in \mathbb{R}^{n}$ is the state vector, $\sigma$ is a function called switching law with domain being a subset of the real numbers, or a subset of the space state or both with image $\mathcal{P}=\{1,2, \cdots, N\}$ where $N$ is the number of subsystems and $f_{p}$ is a complete $\mathcal{C}^{1}$ vector field of $\mathbb{R}^{n}$ for every $p \in \mathcal{P}$. When convenient, arguments of $x(t)$ will be omitted. Let $\sigma: \mathcal{I} \times X \rightarrow \mathcal{P}$, with $\mathcal{I} \subset \mathbb{R}$ a time interval and $X \subseteq \mathbb{R}^{n}$ a subset of the state space, be a switching law depending on the time and state, which we call mixed switching signal and in the case the switching signal is only state dependent 
we simply write $\sigma(x)$. We call $\varphi_{\sigma}\left(t, x_{0}\right)$ the solution of (1) starting at $x_{0}$ at time $t=0$ under the mixed switching law $\sigma$.

Since classical solutions of (1) under a state dependent switching may fail to exist, generalized solutions have been developed. We admit the switching laws are measurable and thus we consider solutions in the sense of Krasowskii (see [25] and references therein for other notions of solutions).

Definition 1. (Krasowskii solution) If $f_{\sigma}(x)$ is a measurable vector field of $\mathbb{R}^{n}$ we say that an absolutely continuous curve $x(t): \mathcal{I} \rightarrow \mathbb{R}^{n}$ is a Krasowskii solution of (1) in the interval $\mathcal{I}$ if it satisfies the differential inclusion

$$
\dot{x}(t) \in K f(x(t))
$$

for $t \in \mathcal{I}$, with $K f(x)=\bigcap_{\delta>0} \overline{c o}\{f(B(x, \delta))\}$ where $f(x)=f_{\sigma}(x), c o\{$.$\} denotes the convex hull and B(x, \delta)$ the open ball of radius $\delta$ and centre $x$.

If $\sigma(x)$ is measurable, then $f_{\sigma}(x)$ is measurable [25]. Thus, for each initial condition $x_{0}$, system (1) admits at least one Krasowskii solution on $t \in\left[0, t_{f}\right)$ with $0<t_{f} \leq+\infty$. Moreover, if the Krasowskii solution is bounded then $t=+\infty$.

We say that a switched solution $\varphi_{\sigma}\left(t, x_{0}\right)$ of (1) is attracted to a compact set $\mathcal{M}$ if for each $\epsilon>0$ there exists a time $T>0$ such that

$$
\varphi_{\sigma}\left(t, x_{0}\right) \in B(\mathcal{M}, \epsilon) \text { for } t \geq T
$$

where $B(\mathcal{M}, \epsilon)=\bigcup_{a \in \mathcal{M}} B(a, \epsilon)$ and $B(a, \epsilon)=\left\{x \in \mathbb{R}^{n}: d(x, a)<\epsilon\right\}$, with $x, a \in \mathbb{R}^{n}$ and $d(x, a)$ a distance function. Equivalently, $\varphi_{\sigma}\left(t, x_{0}\right)$ is attracted to $\mathcal{M}$ if and only if

$$
\lim _{t \rightarrow \infty} d\left(\varphi_{\sigma}\left(t, x_{0}\right), \mathcal{M}\right)=0
$$

\subsection{A stabilizing switching signal}

In this section, a sufficient condition for the existence of a stabilizing switching law for system (1) will be investigated. This condition is given in terms of properties of an auxiliary system, whose vector field is not switched but formed by a convex combination of all vector fields $f_{p}, p \in \mathcal{P}$ of the subsystems of the switched system (1). To this end, consider the set

$$
M=\left\{\alpha \in \mathbb{R}^{N}: \alpha_{p} \geq 0, \forall p \in \mathcal{P} \text { and } \sum_{p=1}^{N} \alpha_{p}=1\right\}
$$

with $\alpha=\left(\alpha_{1} \cdots \alpha_{N}\right)^{\prime}$ and constants $\alpha_{p}$ defining the convex combination of the subsystems $f_{p}$ for all $p \in \mathcal{P}$. We thus consider the auxiliary system of the form

$$
\dot{x}=f(x, \alpha):=\sum_{p=1}^{N} \alpha_{p} f_{p}(x)
$$

where $f(x, \alpha)$ depends on the choice of $\alpha \in M$, and a $\mathcal{C}^{1}$ function $V: \mathbb{R}^{n} \rightarrow \mathbb{R}$. Note that system (5) is not a switched system but a family of continuous dynamic systems with a vector field that is a convex combination of the vector fields of the subsystems of (1).

Proposition 1. Let $\mathcal{B}$ be a compact subset of $\mathbb{R}^{n}$. Suppose the existence of a function $V: \mathbb{R}^{n} \rightarrow \mathbb{R}$ and $\alpha \in M$ such that

$$
\nabla V(x)\left[\sum_{p=1}^{N} \alpha_{p} f_{p}(x)\right]<0
$$


for all $x \in \mathcal{B}$. Then, there exists a state dependent switching law $\sigma(x)$ such that for every switched solution $\varphi_{\sigma}\left(t, x_{0}\right)$ of $(1)$, if $x(t) \in \mathcal{B}$ for all $t \in\left[t_{0}, t_{f}\right]$, then $V(x(t))$ is a decreasing function on $\left[t_{0}, t_{f}\right]$.

Proof. Let $V$ be a $\mathcal{C}^{1}$ function and consider $\alpha \in M$ such that (6) is satisfied. Then, for each $x(t) \in \mathcal{B}$ there exists at least one $p \in \mathcal{P}$ such that

$$
\nabla V(x) f_{p}(x)<0 .
$$

Therefore, there exists a choice for a switching law $\sigma(x)$ such that $\nabla V(x) f_{\sigma}(x)<0$. Since $\mathcal{B}$ is compact, then there exists $\eta>0$ such that

$$
\nabla V(x) f_{\sigma}(x)<-\eta, \quad \forall x \in \mathcal{B}
$$

and function $V$ decreases along the switched solution of system (1) while $\varphi_{\sigma}\left(t, x_{0}\right) \in \mathcal{B}$. As in [26], number $\eta$ can be found by solving $-\eta=\max _{x \in \mathcal{B}} \min _{p \in \mathcal{P}} \nabla V(x) f_{p}(x)$.

Proposition 1 differs from the results given in [7] which ensure that a stabilizing switching signal $\sigma(x)$ can be designed for the case $\mathcal{B}=\mathbb{R}^{n}$. Proposition 2 following offers one choice of a switching law that ensures $V(x(t)$ is a decreasing function while $x(t) \in \mathcal{B}$.

Proposition 2. A switching law given by

$$
\sigma(x)=\left\{\begin{array}{cc}
1, & \text { if } \quad x \in \Gamma_{1} \\
p, & \text { if } \quad x \in\left(\Gamma_{p} \backslash\left(\bigcup_{k<p} \Gamma_{k}\right)\right)
\end{array}\right.
$$

where $\Gamma_{p}=\left\{x \in \mathcal{B}: \nabla V(x) f_{p}(x)<0\right.$ and $\left.\nabla V(x) f_{p}(x) \leq \nabla V(x) f_{k}(x), \forall k \in \mathcal{P}-\{p\}\right\}$ satisfies Proposition 1 and is measurable.

Proof. The switching law given by (9) satisfies Proposition 1 due to the construction of sets $\Gamma_{p}$. Thus, the switching law given by Propositions 2 ensures that

$$
\nabla V(x) f_{\sigma}(x)<-\eta, \quad \forall x \in \mathcal{B}
$$

which guarantees $V(x)$ decreases along the switched solution of (1).

Now, we will prove that the switching law (9) is measurable. Consider $\sigma: \mathcal{B} \rightarrow \mathcal{P}, H_{p}=\{x \in \mathcal{B}: \sigma(x)=p\}$ which satisfies $H_{i} \cap H_{j}=\emptyset$ for any $i \neq j \in \mathcal{P}$ and $\bigcup_{p \in \mathcal{P}} H_{p}=\mathcal{B}$. Let the Borel $\sigma$-algebra $\Sigma_{1}$ and $\Sigma_{2}$ be formed by subsets of $\mathcal{B}$ and $\mathcal{P}$, respectively, then

$$
\sigma^{-1}(p)=\Gamma_{p} \backslash\left(\bigcup_{k<p} \Gamma_{k}\right)=H_{p}, \quad \forall p \in \Sigma_{2}
$$

Since $\Gamma_{p} \in \Sigma_{1}$ and $H_{p}=\left(\bigcup_{k<p} \Gamma_{k}\right)^{c} \bigcap \Gamma_{p} \in \Sigma_{1}$ for each $k$ and $p$ where the first term of the equality is the complementary of set $\left(\bigcup_{k<p} \Gamma_{k}\right)$, the switching signal $\sigma(x)$ is a measurable function.

Therefore, by Definition 1 , for all $x_{0} \in \mathcal{B}$ there exists at least a Krasowskii solution of (1) such that $V$ decreases along this solution while $\varphi_{\sigma}\left(t, x_{0}\right) \in \mathcal{B}$.

Corollary 1. If $\mathcal{B}=\mathbb{R}^{n}$ in Propositions 1 and 2, then the switching law $\sigma(x)$ is a stabilizing one, that is, all the solutions tend to the origin as $t \rightarrow \infty$. 


\section{Ultimately bounded solutions of switched systems}

In this section, we explore the results of Section 2 and invariance properties of level sets of an auxiliary scalar function $V$ to prove that solutions of the switched system (1) are ultimately bounded under the switching law given in Proposition 2. To this end, we admit a measurable switching law outside a level set of an auxiliary function $V$ and the switching signal can be designed to guarantee the solutions will enter this level set. However, inside this level set, there can be no choice of switching signal that can guarantee $V$ will be a decreasing function. In spite of that, using an arbitrary but a measurable switching law inside of this level set of $V$, we guarantee the solutions will be ultimately bounded.

Consider $V: \mathbb{R}^{n} \rightarrow \mathbb{R}$ and assume the level set

$$
\Omega_{c}=\left\{x \in \mathbb{R}^{n}: V(x)<c\right\}
$$

is bounded. Define the compact set $\Omega_{L, \ell}=\bar{\Omega}_{L} \backslash \Omega_{\ell}$. Also, define the following set:

$$
C_{\alpha}=\left\{x \in \mathbb{R}^{n}: \nabla V(x) f(x, \alpha)>0\right\} .
$$

Set $C_{\alpha}$ denotes the set in $\mathbb{R}^{n}$ where the derivative of the scalar function $V$ along the solution of the auxiliary system (5) is positive. Lemma 1 explores the auxiliary system (5) and a scalar function $V$ associated with (5) to study the asymptotic behaviour of solutions of the switched system (1).

Lemma 1. Consider the switched system (1) and let $V: \mathbb{R}^{n} \rightarrow \mathbb{R}$ be a $\mathcal{C}^{1}$ function. Let $\alpha \in M, \ell, L$ be real numbers satisfying $\sup _{x \in C_{\alpha}} V(x)<\ell<L<\infty$ and suppose that $\Omega_{L}$ is bounded. If $x_{0} \in \Omega_{L}$ then there exists a mixed measurable switching law $\sigma(t, x)$, obeying the switching law of Proposition 2 in the set $\Omega_{L, \ell}$, such that:

(i) the solution $\varphi_{\sigma}\left(t, x_{0}\right) \in \Omega_{L}, \forall t \geq 0$

(ii) there exists $T \geq 0$ such that $\varphi_{\sigma}\left(t, x_{0}\right) \in \bar{\Omega}_{\ell}, \forall t>T$.

Proof. (i) Consider $x_{0} \in \Omega_{L}$ such that $x_{0} \notin \Omega_{\ell}$. Since set $C_{\alpha} \subset \Omega_{\ell}$ then, while the solution $\varphi_{\sigma}\left(t, x_{0}\right) \in \Omega_{L, \ell}$, inequality (6) is satisfied and for this a measurable switching law can be chosen according to the state dependent switching signal given in Proposition 2. This choice of switching law ensures that function $V$ decreases along the Krasowskii solution of (1) for all $t \geq 0$ while $x \in \Omega_{L, \ell}$. Moreover, since $\Omega_{L, \ell}$ is a compact set, then there exists $\eta>0$ such that $-\eta=\max _{x \in \Omega_{L, \ell}} \min _{p \in \mathcal{P}} \nabla V(x) f_{p}(x)$. Thus, the switching law of Proposition 2 ensures that $\nabla V(x) f_{\sigma}(x)<-\eta, \forall x \in \Omega_{L, \ell}$, which guarantees not only that $\varphi\left(t, x_{0}\right)$ stays inside $\Omega_{L}$ for all $t \geq 0$, but also ensures the existence of a finite time $T \geq 0$ such that $V\left(\varphi_{\sigma}\left(T, x_{0}\right)\right)<\ell$. (ii) Start the proof assuming $x_{1}=\varphi_{\sigma}\left(T, x_{0}\right) \in \Omega_{\ell}$. Suppose the existence of a time instant $\tilde{t}>T$ such that $\varphi_{\sigma}\left(\tilde{t}, x_{0}\right) \notin \bar{\Omega}_{\ell}$. Then, by the continuity of $V$ and $\varphi\left(t, x_{0}\right)$ and the fact that $V\left(\varphi\left(t, x_{0}\right)\right)>\ell$, $\forall t \in[\bar{t}, \tilde{t}]$ there exists $\bar{t} \in(T, \tilde{t})$ such that $V\left(\varphi\left(\bar{t}, x_{0}\right)\right)=\ell$. Since Proposition 2 is satisfied outside of $\Omega_{\ell}$ then $V$ decreases along the switched solution of system (1) in $\Omega_{L, \ell}$ leading to a contradiction, thus $\varphi_{\sigma}\left(t, x_{0}\right) \in \bar{\Omega}_{\ell}$ for all $t \geq T$ and the proof is complete.

In Lemma 1, sets $\Omega_{\ell}$ and $\Omega_{L}$, respectively, are estimates of the attractor and basin of attraction in the sense that the attractor is contained in $\Omega_{\ell}$ and $\Omega_{L}$ is entirely contained in the attraction domain. Lemma 1 admits that a suitable choice of a switching law can be done outside $\Omega_{\ell}$ to guarantee the solutions are ultimately bounded. Inside $\Omega_{\ell}$ dissipativeness cannot be guaranteed and a stabilizing switching law may not exist but a measurable switching signal is sufficient to guarantee that the solutions exist and do not leave $\bar{\Omega}_{\ell}$.

\subsection{Switched TS fuzzy systems}

A TS fuzzy model is based on a set of fuzzy rules to describe a nonlinear system in terms of a set of local linear models which are smoothly connected by fuzzy membership functions. This modelling is able to approximate any smooth nonlinear functions to any degree of accuracy in a convex compact region [8]. Moreover, TS fuzzy modelling 
drastically reduces the number of fuzzy rules to describe complex nonlinear systems than conventional fuzzy techniques [10] and provides a basis for development of systematic approaches to stability analysis and controller design in the framework of LMIs which can be efficiently solved by convex programming techniques [27].

In this section, a TS fuzzy modelling is used to obtain sufficient LMI-based conditions for the existence of an auxiliary function $V(x)$ and constants $\alpha, \ell$ and $L$ satisfying the conditions of Lemma 1 and therefore ensuring the solution is attracted to an invariant set.

The results of this section consider a subclass of the subsystems appearing in (1) given by:

$$
\dot{x}(t)=F_{p}(x(t)) x(t)
$$

where $x(t) \in \mathbb{R}^{n}$ is the state vector, $F_{p} \in \mathbb{R}^{n \times n}$ is a matrix of nonlinear functions, for all $p \in \mathcal{P}$. Here, the nonlinear system (14) is exactly represented by a TS fuzzy model using the concept of sector non-linearities, as in [8,28], in the following subset of the state space

$$
S_{p}:=\left\{x \in \mathbb{R}^{n}:\left|x_{v}\right| \leq \bar{x}_{p v}, v \in \mathcal{T} \text { and } p \in \mathcal{P}\right\}
$$

where $\mathcal{T}=\{1,2, \cdots, q\}, q \leq n$, and $\bar{x}_{p v}$ is a positive real number for all $v \in \mathcal{T}, p \in \mathcal{P}$.

The blending of the models of the subsystem $p$ of the switched nonlinear system (14) can be described as:

$$
\dot{x}(t)=\sum_{k \in \mathcal{R}_{p}} h_{p k}(x(t)) \mathbf{A}_{p k} x(t)
$$

where $h_{p k}(x(t))$ are nonlinear functions with $\mathcal{R}_{p}=\left\{1,2, \cdots, 2^{r_{p}}\right\}$ with $A_{p k}$ the matrices of the local linear system (14) in the set $S_{p}, \forall p \in \mathcal{P}$ and $r_{p}$ is the number of the non-constant terms in $F_{p}$.

Remark 1. If no constraints on the state are needed for some $p$, then $S_{p}=\mathbb{R}^{n}$.

From the properties of membership functions we have:

$$
h_{p k}(x(t)) \geq 0 \text { and } \sum_{k \in \mathcal{R}_{p}} h_{p k}(x(t))=1
$$

for all $p \in \mathcal{P}$ and $k \in \mathcal{R}_{p}$. Using (17), it follows that

$$
\left(\sum_{k \in \mathcal{R}_{\beta}} h_{\beta k}(x(t))-\frac{1}{N-1} \sum_{\substack{p \in \mathcal{P} \\ p \neq \beta}} \sum_{k \in \mathcal{R}_{p}} h_{p k}(x(t))\right)\left(\sum_{p \in \mathcal{P}} \sum_{k \in \mathcal{G}_{p}} h_{p k}\right)=0
$$

with $\beta \in \mathcal{P}$. When convenient, arguments of $h_{p k}(x(t))$ will be omitted.

\subsection{Main results}

Suppose system (1) can be written in the form of (14). Now, if we represent each subsystem in (14) by a TS fuzzy model (16), we have that function (5) assumes the following form:

$$
f(x, \alpha)=\sum_{p \in \mathcal{P}} \alpha_{p}\left(\sum_{k \in \mathcal{R}_{p}} h_{p k} \mathbf{A}_{p k}\right) x .
$$

Define the following sets:

$$
\begin{aligned}
Z & =\left\{x \in \mathbb{R}^{n}:\left|x_{v}\right| \leq \bar{x}_{v}, \forall v \in\{1,2, \ldots, n\} \text { where } \bar{x}_{v}=\min _{p \in \mathcal{P}} \bar{x}_{p v}, \forall v \in \mathcal{T}\right\} \\
Z_{v} & =\left\{x \in Z: e_{v}^{\prime} x=\bar{x}_{v}\right\} \bigcup\left\{x \in Z: e_{v}^{\prime} x=-\bar{x}_{v}\right\}, \forall v \in\{1,2, \cdots, n\} \\
\Omega_{\ell} & =\{x \in Z: V(x)<\ell\} \text { and } \\
\mathcal{D} & =\left\{x \in Z: x^{\prime}\left[\sum_{p \in \mathcal{P}} \sum_{k \in \mathcal{G}_{p}} \dot{h}_{p k}(x) P_{p k}\right] x>0\right\}, \text { with } \dot{h}_{p k}(x)=\nabla h_{p k}(x) f(x, \alpha) .
\end{aligned}
$$


The next results are developed using a scalar non-quadratic function $V: Z \rightarrow \mathbb{R}$ given by:

$$
V(x)=x^{\prime} P_{z}(x) x
$$

where

$$
P_{z}(x)=\sum_{p \in \mathcal{P}} \sum_{k \in \mathcal{G}_{p}} h_{p k} \mathbf{P}_{p k},
$$

and $\mathcal{G}_{p}$ is a subset of $\mathcal{R}_{p}$ for all $p \in \mathcal{P}$, which is previously chosen. When convenient, arguments of $P_{z}(x)$ will be omitted.

Local stability of fuzzy systems under a non-quadratic Lyapunov function has been studied in $[9,14,15]$. The main drawback of these results is that the LMIs conditions for stability are obtained imposing bounds on the membership functions time derivatives, such as $\left|\dot{h}_{p k}\right| \leq \phi_{p k}, p \in \mathcal{P}, k \in \mathcal{R}_{p}$ with $\phi_{p k}$ design parameters. As a consequence, the choice of $\phi_{p k}, p \in \mathcal{P}, k \in \mathcal{R}_{p}$ affects the estimates of the attraction domain of TS fuzzy systems. To overcome this drawback, in [16,17] properties of membership functions were explored to obtain less conservative LMI conditions for asymptotic stability of TS fuzzy systems.

Here, the problem related with the bounds on the membership functions time derivative was converted to the problem of finding a positively invariant set $\Omega_{\ell}$ which contains $\mathcal{D}$ for system (16). The results explores properties of membership functions to obtain a scalar function $V$ which can attain positive time derivative in some sets, relaxing the stability conditions of Lyapunov theory. In this case, it is only necessary to verify if the set where the time derivative of function $V$ assume positive values is bounded. The LMI conditions use a matrix transformation due to [29] and the Finsler Lemma to decouple the matrices of the auxiliary function (24) from the fuzzy system matrices, reducing the conservativeness on the numerical solution [9].

Theorem 1. Consider the switched system (1) with $f_{p}$ described by the TS fuzzy model (16) in set Z. Iffor given $\mu>0$ and $\alpha \in M$, there exist matrices $\mathbf{P}_{p k}=\mathbf{P}_{p k}^{\prime} \in \mathbb{R}^{n \times n}, \mathbf{M} \in \mathbb{R}^{2 n \times 2 n}, \mathbf{L}_{p k} \in \mathbb{R}^{n \times n}, \mathbf{R}_{p k} \in \mathbb{R}^{n \times n}$ satisfying (26)-(35) and real numbers $\ell, a>0$ such that, $\sup _{x \in \mathcal{D}} V(x)<\ell<a<\min _{x \in \partial Z} \sum_{p \in \mathcal{P}} \sum_{k \in \mathcal{G}_{p}} h_{p k}$. Then, every solution $\varphi_{\sigma}\left(t, x_{0}\right)$ of $(1)$, with $x_{0} \in \Omega_{a}$, possessing a mixed switching law $\sigma(t, x)$ obeying Proposition 2 while $x(t) \in \Omega_{a, \ell}$ is attracted to $\bar{\Omega}_{\ell}$. Moreover, $\Omega_{a}$ can be enlarged as close as possible to the boundary of set $Z$.

$$
\begin{aligned}
& \Upsilon_{\beta k_{-} \beta k}+\mathbf{Q} \prec \mathbf{0}, \quad k \in \mathcal{G}_{\beta} \\
& \Upsilon_{\beta k_{-} i j}+\mathbf{Q} \prec \mathbf{0}, \quad k \in \mathcal{R}_{\beta}-\mathcal{G}_{\beta}, \quad i \in \mathcal{P}, \quad j \in \mathcal{G}_{i} \\
& \Upsilon_{\beta k_{-} \beta j}+\Upsilon_{\beta j \_\beta k}+2 \mathbf{Q} \prec \mathbf{0}, \quad j, k \in \mathcal{G}_{\beta}, \quad j<k \\
& \Upsilon_{p k_{-} p k}-\frac{1}{N-1} \mathbf{Q} \prec \mathbf{0}, \quad p \in \mathcal{P}-\{\beta\}, \quad k \in \mathcal{G}_{p} \\
& \Upsilon_{p k_{-} i j}-\frac{1}{N-1} \mathbf{Q} \prec \mathbf{0}, \quad p \in \mathcal{P}-\{\beta\}, \quad k \in \mathcal{R}_{p}-\mathcal{G}_{p}, \quad i \in \mathcal{P}, \quad j \in \mathcal{G}_{i} \\
& \Upsilon_{p k_{-} p j}+\Upsilon_{p j_{-} p k}-\frac{2}{N-1} \mathbf{Q} \prec \mathbf{0}, \quad p \in \mathcal{P}-\{\beta\}, \quad k, j \in \mathcal{G}_{p}, \quad j<k \\
& \Upsilon_{p k_{-} i j}+\Upsilon_{i j_{-} p k}-\frac{2}{N-1} \mathbf{Q} \prec \mathbf{0}, \quad p, i \in \mathcal{P}-\{\beta\}, \quad p<i, \quad k \in \mathcal{G}_{p}, \quad j \in \mathcal{G}_{i} \\
& \Upsilon_{\beta k_{-} i j}+\Upsilon_{i j j_{-} \beta k}+\frac{N-2}{N-1} \mathbf{Q} \prec \mathbf{0}, \quad k \in \mathcal{G}_{\beta}, \quad i \in \mathcal{P}-\{\beta\}, \quad j \in \mathcal{G}_{i} \\
& \frac{1}{\bar{x}_{v}^{2}} e_{v} e_{v}^{\prime}<P_{p k}, \forall v \in\{1,2, \ldots, n\}, p \in \mathcal{P}, k \in \mathcal{G}_{p} \\
& P_{p k} \leq \mu I, \quad \forall p \in \mathcal{P}, k \in \mathcal{G}_{p},
\end{aligned}
$$

where $\mathbf{Q}=\sum_{p \in \mathcal{P}} \sum_{k \in\left(\mathcal{R}_{p}-\mathcal{G}_{p}\right)} \Upsilon_{p k_{-} p k}+\mathbf{M}$ and $\Upsilon_{p k_{-} i j}=\left[\begin{array}{cc}\alpha_{i}\left(\mathbf{L}_{p k} \mathbf{A}_{i j}+\mathbf{A}_{i j}^{\prime} \mathbf{L}_{p k}^{\prime}\right) & \star \\ \mathbf{P}_{p k}-\mathbf{L}_{p k}^{\prime}+\alpha_{i} \mathbf{R}_{p k} \mathbf{A}_{i j} & -\mathbf{R}_{p k}-\mathbf{R}_{p k}^{\prime}\end{array}\right]$. 
Proof. Following the same idea of [30], if (34) is feasible then

$$
\sum_{p \in \mathcal{P}} \sum_{k \in \mathcal{G}_{p}} h_{p k} \frac{1}{\bar{x}_{v}^{2}} e_{v} e_{v}^{\prime}<P_{z}, \forall x \in Z, v \in\{1,2, \ldots, n\}
$$

Since $a<\min _{x \in \partial Z} \sum_{p \in \mathcal{P}} \sum_{k \in \mathcal{G}_{p}} h_{p k}$, from (36) and the definition of $Z_{v}$ we have that $\bigcup_{v \in\{1,2, \ldots, n\}} Z_{v}=\partial Z$ and

$$
V(x)=x^{T} P_{z} x>\sum_{p \in \mathcal{P}} \sum_{k \in \mathcal{G}_{p}} h_{p k} x^{T}\left(\frac{1}{\bar{x}_{v}^{2}} e_{v} e_{v}^{\prime}\right) x=\sum_{p \in \mathcal{P}} \sum_{k \in \mathcal{G}_{p}} h_{p k} \frac{\bar{x}_{v}^{2}}{\bar{x}_{v}^{2}}=\sum_{p \in \mathcal{P}} \sum_{k \in \mathcal{G}_{p}} h_{p k}>a, \quad \forall x \in Z_{v} .
$$

Then, for all $x \in \partial Z, V(x) \notin \Omega_{a}$, thus the level set $\Omega_{a}$ is contained in $Z$ and $\partial \Omega_{a} \cap \partial Z=\emptyset$.

Now, if (26)-(33) are feasible with $\alpha \in M$, then multiplying (26) by $h_{\beta k}^{2}$, (27) by $h_{\beta k} h_{i j}$, (28) by $h_{\beta k} h_{\beta j}$, (29) by $h_{p k}^{2}$, (30) and (32) by $h_{p k} h_{i j}$, (31) by $h_{p k} h_{p j}$, (33) by $h_{\beta k} h_{i j}$ and adding all terms we obtain:

$$
\begin{aligned}
& \sum_{k \in\left(\mathcal{R}_{\beta}-\mathcal{G}_{\beta}\right)} \sum_{i \in \mathcal{P}} \sum_{j \in \mathcal{G}_{i}} h_{\beta k} h_{i j}\left(\Upsilon_{\beta k_{-} i j}+\mathbf{Q}\right)+\sum_{k \in \mathcal{G}_{\beta}} \sum_{\substack{j \in \mathcal{G}_{\beta} \\
j<k}} h_{\beta k} h_{\beta j}\left(\Upsilon_{\beta k_{-} \beta j}+\Upsilon_{\beta j \_\beta k}+2 \mathbf{Q}\right) \\
& +\sum_{\substack{p \in \mathcal{P} \\
p \neq \beta}} \sum_{k \in \mathcal{G}_{p}} h_{p k}^{2}\left(\Upsilon_{p k_{-} p k}-\frac{1}{N-1} \mathbf{Q}\right)+\sum_{\substack{p \in \mathcal{P} \\
p \neq \beta}} \sum_{k \in\left(\mathcal{R}_{p}-\mathcal{G}_{p}\right)} \sum_{i \in \mathcal{P}} \sum_{j \in \mathcal{G}_{i}} h_{p k} h_{i j}\left(\Upsilon_{p k_{-} i j}-\frac{1}{N-1} \mathbf{Q}\right)
\end{aligned}
$$

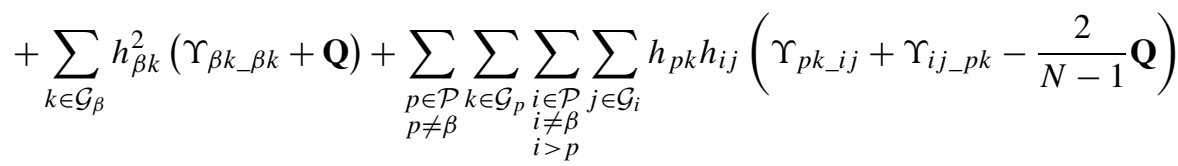

$$
\begin{aligned}
& +\sum_{\substack{p \in \mathcal{P} \\
p \neq \beta}} \sum_{k \in \mathcal{G}_{p}} \sum_{\substack{j \in \mathcal{G}_{p} \\
j<k}} h_{p k} h_{p j}\left(\Upsilon_{p k_{-} p j}+\Upsilon_{p j_{-} p k}-\frac{2}{N-1} \mathbf{Q}\right) \\
& +\sum_{k \in \mathcal{G}_{\beta}} \sum_{\substack{i \in \mathcal{P} \\
i \neq \beta}} \sum_{j \in \mathcal{G}_{i}} h_{\beta k} h_{i j}\left(\Upsilon_{\beta k \_i j}+\Upsilon_{i j \_\beta k}+\frac{N-2}{N-1} \mathbf{Q}\right) \\
& =\sum_{p \in \mathcal{P}} \sum_{k \in \mathcal{G}_{p}} \sum_{i \in \mathcal{P}} \sum_{j \in \mathcal{G}_{i}} h_{p k} h_{i j} \Upsilon_{p k} i j+\left(\sum_{k \in \mathcal{R}_{\beta}} h_{\beta k}-\frac{1}{N-1} \sum_{\substack{p \in \mathcal{P} \\
p \neq \beta}} \sum_{k \in \mathcal{R}_{p}} h_{p k}\right)\left(\sum_{p \in \mathcal{P}} \sum_{k \in \mathcal{G}_{p}} h_{p k}\right) \mathbf{Q} \prec 0 .
\end{aligned}
$$

Replacing (18) in (37) we have that

$$
\left[\begin{array}{lc}
\mathbf{L}(h) \mathbf{A}(\alpha, h)+\mathbf{A}(\alpha, h)^{\prime} \mathbf{L}(h)^{\prime} & \star \\
\mathbf{P}(h)-\mathbf{L}(h)^{\prime}+\mathbf{R}(h) \mathbf{A}(\alpha, h) & -\mathbf{R}(h)-\mathbf{R}(h)^{\prime}
\end{array}\right] \prec \mathbf{0}
$$

where $\mathbf{L}(h)=\sum_{p \in \mathcal{P}} \sum_{k \in \mathcal{G}_{p}} h_{p k} \mathbf{L}_{p k}, \mathbf{R}(h)=\sum_{p \in \mathcal{P}} \sum_{k \in \mathcal{G}_{p}} h_{p k} \mathbf{R}_{p k}$ and $\mathbf{A}(\alpha, h)=\sum_{p \in \mathcal{P}} \sum_{k \in \mathcal{R}_{p}} \alpha_{p} h_{p k} \mathbf{A}_{p k}$. Pre-multiplying and post-multiplying (38) by the vector $\left[x^{\prime} x^{\prime} \mathbf{A}(\alpha, h)^{\prime}\right]$ and its transpose, respectively, it yields

$$
x^{\prime}\left\{\mathbf{A}(\alpha, h)^{\prime} \mathbf{P}(h)+\mathbf{P}(h) \mathbf{A}(\alpha, h)\right\} x \prec \mathbf{0} .
$$

The time-derivative of function (24) along the trajectories of (19) is given by

$$
\nabla V(x) f(x, \alpha)=x^{\prime}\left\{\sum_{p \in \mathcal{P}} \sum_{k \in \mathcal{G}_{p}} \dot{h}_{p k} \mathbf{P}_{p k}\right\} x+x^{\prime}\left\{\mathbf{A}(\alpha, h)^{\prime} \mathbf{P}(h)+\mathbf{P}(h) \mathbf{A}(\alpha, h)\right\} x .
$$


From (39) we have that the second part of (40) is negative, then we conclude that $x^{\prime}\left\{\sum_{p \in \mathcal{P}} \sum_{k \in \mathcal{G}_{p}} \dot{h}_{p k} \mathbf{P}_{p k}\right\} x$ is the only term that can make (40) positive and we conclude that the set $C_{\alpha}$ for $x \in Z$ is contained in $\mathcal{D}$, that is, $C_{\alpha} \subseteq \mathcal{D}$. Since $\ell<a$, that is, $\mathcal{D} \subset \Omega_{\ell}$, by Lemma 1 every solution $\varphi_{\sigma}\left(t, x_{0}\right)$ of (1) with $x_{0} \in \Omega_{a}$ possessing a measurable mixed switching law satisfying Proposition 2 for all $t>0$ while $x(t) \in \Omega_{a, \ell}$ is attracted to $\Omega_{\ell}$. Moreover, if (35) holds, then $x^{\prime} \sum_{p \in \mathcal{P}} \sum_{k \in \mathcal{G}_{p}} h_{p k} P_{p k} x \leq \sum_{p \in \mathcal{P}} \sum_{k \in \mathcal{G}_{p}} h_{p k} x^{\prime} \mu x$. Therefore, the set $\left\{x \in \mathbb{Z}: x^{\prime} x \leq \frac{a}{M \mu}\right\} \subseteq \Omega_{a}$ with $M=\max _{x \in Z} \sum_{p \in \mathcal{P}} \sum_{k \in \mathcal{G}_{p}} h_{p k}$, and thus minimizing $\mu$ makes $\Omega_{a}$ to be maximized. Since $\Omega_{a} \subset Z$, the boundary of $\Omega_{a}$ is enlarged as close as possible to the boundary of $Z$.

Observing (16) we verify that the origin is a common equilibrium point shared by all subsystems $p \in \mathcal{P}$. In spite of that, it might be difficult, in some cases to prove asymptotically stability due to the presence of subsystems that have ultimately bounded solutions. Theorem 1 relaxes the negativeness property of the derivative of scalar function $V$ to facilitate the search for a solution of the LMIs. As a trade-off, we do not prove stability of the equilibrium but that the solutions are ultimately bounded and converge to a bounded set.

\subsection{Example}

In this example, we find an auxiliary function for a switched system using Theorem 1 and an estimate of a positively invariant set for the solutions. We consider a switched system with $\mathcal{P}=\{1,2\}$ and

$$
f_{1}(x)=\left[\begin{array}{c}
\frac{x_{1}}{50}\left(-6 x_{1}^{2}-6 x_{2}^{2}+50\right) \\
x_{2}
\end{array}\right], \quad f_{2}(x)=\left[\begin{array}{c}
-10 x_{1}+\frac{30}{25} x_{2}^{3}-15 x_{2} \\
-3 x_{2}
\end{array}\right] .
$$

The solution of subsystem $p=1$ of the switched system is not ultimately bounded. Instead of searching for a candidate Lyapunov-like function satisfying the hypothesis of Lemma 1, we explore the systematic procedure given by Theorem 1 to analyse the solution of a switched system with $f_{1}(x)$ and $f_{2}(x)$ given by (41). For this, let $f_{1}(x)$ and $f_{2}(x)$ be locally described as a TS fuzzy model [8]. Therefore, the switched nonlinear subsystems is described as the overall fuzzy subsystem (16) with local models:

$$
\begin{aligned}
& \mathbf{A}_{11}=\left[\begin{array}{ll}
1 & 0 \\
0 & 1
\end{array}\right], \quad \mathbf{A}_{12}=\left[\begin{array}{rr}
-5 & 0 \\
0 & 1
\end{array}\right] \\
& \mathbf{A}_{21}=\left[\begin{array}{rr}
-10 & 15 \\
0 & -3
\end{array}\right], \quad \mathbf{A}_{22}=\left[\begin{array}{rr}
-10 & -15 \\
0 & -3
\end{array}\right],
\end{aligned}
$$

and membership functions

$$
\begin{aligned}
& h_{11}=\frac{50-x_{1}^{2}-x_{2}^{2}}{50}, h_{12}=1-h_{11} \\
& h_{21}=\frac{x_{2}^{2}}{25}, h_{22}=1-h_{21}
\end{aligned}
$$

in the set $Z=\left\{x \in \mathbb{R}^{2}:\left|x_{1}\right| \leq 5\right.$ and $\left.\left|x_{2}\right| \leq 5\right\}$. Let $\mathcal{G}_{1}=\{2\}$ and $\mathcal{G}_{2}=\{1\}$, using MATLAB toolboxes YALMIP [31] and SeDuMi [27] to solve (26)-(35) with fixed parameters $\alpha_{1}=0.6, \alpha_{2}=0.4, \beta=1$ and $\mu=0.19$ for all $p \in \mathcal{P}$ and $k \in \mathcal{R}_{p}$, we obtain the following matrices:

$$
\begin{aligned}
& \mathbf{P}_{11}=\left[\begin{array}{ll}
-0.0069 & -0.0020 \\
-0.0020 & -0.0526
\end{array}\right], \mathbf{P}_{12}=\left[\begin{array}{ll}
0.0413 & 0.0075 \\
0.0075 & 0.1820
\end{array}\right] \\
& \mathbf{P}_{21}=\left[\begin{array}{ll}
0.0403 & 0.0000 \\
0.0000 & 0.1876
\end{array}\right], \mathbf{P}_{22} \approx 0 .
\end{aligned}
$$

Set $\mathcal{D}$ is given by

$$
\mathcal{D}=\left\{x \in Z: x^{\prime}\left[\dot{h}_{12}(x) P_{12}+\dot{h}_{21}(x) P_{21}\right] x>0\right\}
$$




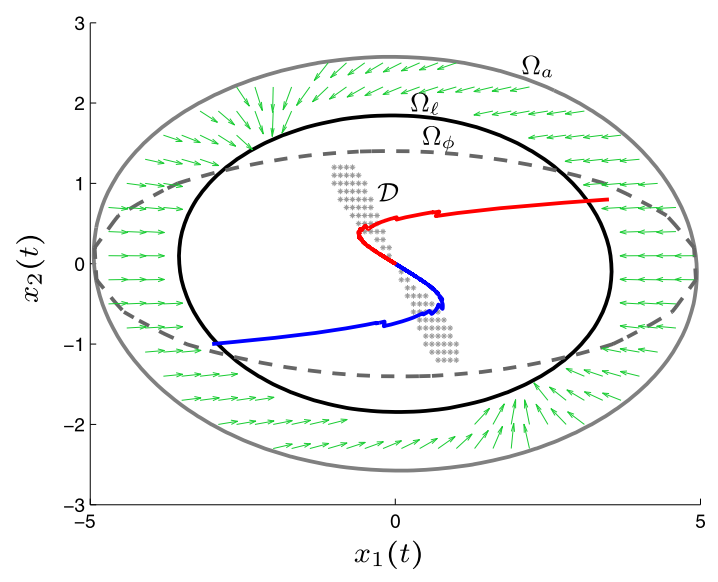

Fig. 1. Phase portrait with $x_{0_{1}}=\left[\begin{array}{ll}-3 & -1\end{array}\right]$ and $x_{0_{2}}=\left[\begin{array}{ll}3.5 & 0.8\end{array}\right]$ illustrating the level sets $\Omega_{a}, \Omega_{\ell}$ and the region $\Omega_{\phi}$ obtained with LMIs established with bounds on the membership functions.

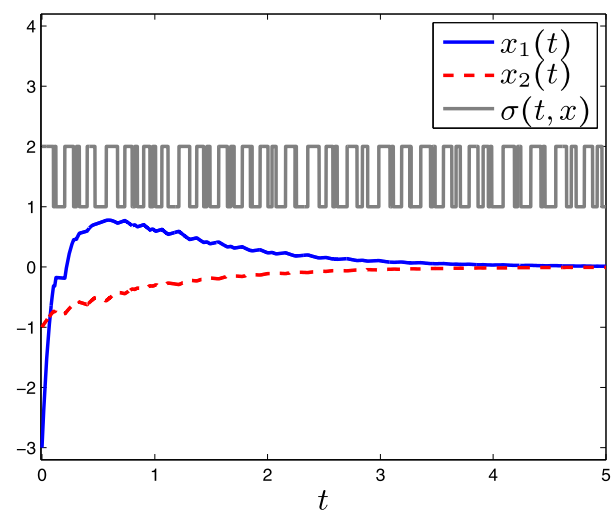

Fig. 2. Switching solution and switching signal $\sigma(t, x)$ for initial condition $x_{0}=\left[\begin{array}{ll}-3-1\end{array}\right]$.

with

$$
\begin{aligned}
& \nabla h_{12} f(x, \alpha)=\frac{x_{1}\left[\alpha_{1} \frac{x_{1}}{50}\left(-6 x_{1}^{2}-6 x_{2}^{2}+50\right)+\alpha_{2}\left(-10 x_{1}+\frac{30}{25} x_{2}^{3}-15 x_{2}\right)\right]+x_{2}\left(\alpha_{1} x_{2}-3 \alpha_{2} x_{2}\right)}{25} \\
& \nabla h_{21} f(x, \alpha)=\frac{2 x_{2}\left(\alpha_{1} x_{2}-3 \alpha_{2} x_{2}\right)}{25} .
\end{aligned}
$$

Choosing $\ell=0.13$ and $a=0.491$ such that $\sup _{x \in \mathcal{D}} V(x)<\ell<a<\min _{x \in \partial Z} \sum_{p \in \mathcal{P}} \sum_{k \in \mathcal{G}_{p}} h_{p k}=1 / 2$, Fig. 1 shows sets $\mathcal{D}$ (dotted region), $\Omega_{\ell}, \Omega_{a}$ for $x^{T} x<\frac{a}{2 \mu}, \mu=0.19$, and the switching solution for initial conditions $x_{0_{1}}=[-3-1]$ and $x_{0_{2}}=$ [3.5 0.8]. Thus, by Theorem 1 , every bounded solution $\varphi_{\sigma}\left(t, x_{0}\right)$ of (1) with $x_{0} \in \Omega_{a}$ possessing a mixed switching $\sigma(t, x)$ satisfying Proposition 2, while $x(t) \in \Omega_{a, \ell}$, is attracted to $\bar{\Omega}_{\ell}$. For comparison purposes, we included the area of attraction obtained with the LMIs established in terms of bounds on the membership functions time derivatives given in [23, Theorem 1]. The area of attraction denoted $\Omega_{\phi}$ was obtained considering the bounds $\left|\dot{h}_{p k}\right| \leq 5.1, p=1,2$ and $k=1,2$. Note that in Fig. 1, $\Omega_{\phi} \subset \Omega_{a}$. As a matter of fact, LMIs (34) and (35) succeeded in maximizing the bounded attraction domain in set Z. Figs. 2, 3 and 4 illustrate the time-domain simulation, the mixed switching law $\sigma(t, x)$ and function $V$, respectively, for initial condition $x_{0_{1}}$ while Figs. 5, 6 and 7 show the same for initial condition $x_{0_{2}}$. 


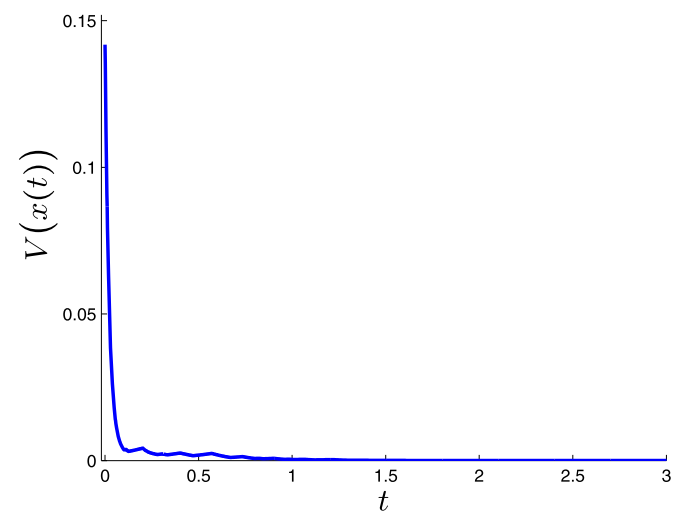

Fig. 3. Function $V$ along the switched system solution with initial condition $x_{0}=\left[\begin{array}{ll}-3-1 & -\end{array}\right.$.

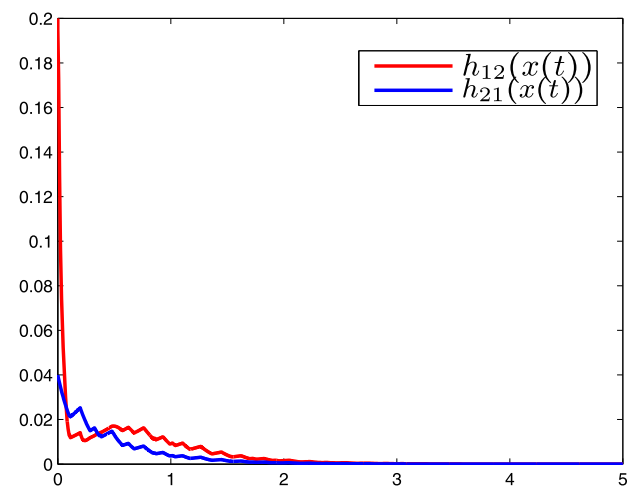

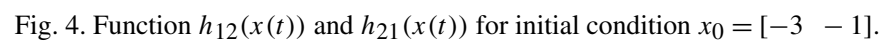

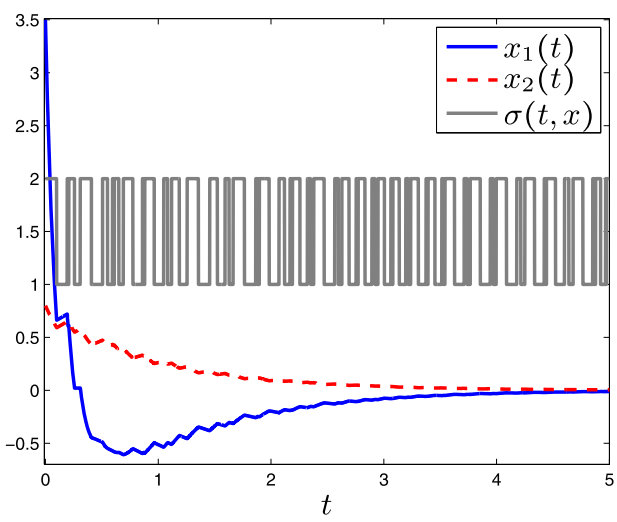

Fig. 5. Switching solution and switching signal $\sigma(t, x)$ for initial condition $x_{0}=\left[\begin{array}{ll}3.5 & 0.8\end{array}\right]$.

Remark 2. Note that, using a fuzzy TS approach, Theorem 1 provides a function $V=x^{\prime}\left(h_{12} P_{12}+h_{21} P_{21}\right) x$, with $h_{12}, h_{21}$ given by (43) and $P_{12}, P_{21}$ given by (44), satisfying the hypothesis of Lemma 1 in the set $Z$ as with $\ell=0.13$ and $L=a=0.491$ we have $C_{\alpha} \subset \mathcal{D} \subset \Omega_{\ell} \subset \Omega_{a}$ for $x \in Z$. Therefore, with the function $V$ found, it is possible to obtain $\Omega_{\ell}$ and $\Omega_{a}$ as estimates of the attractor and area of attraction of switched systems, respectively. 


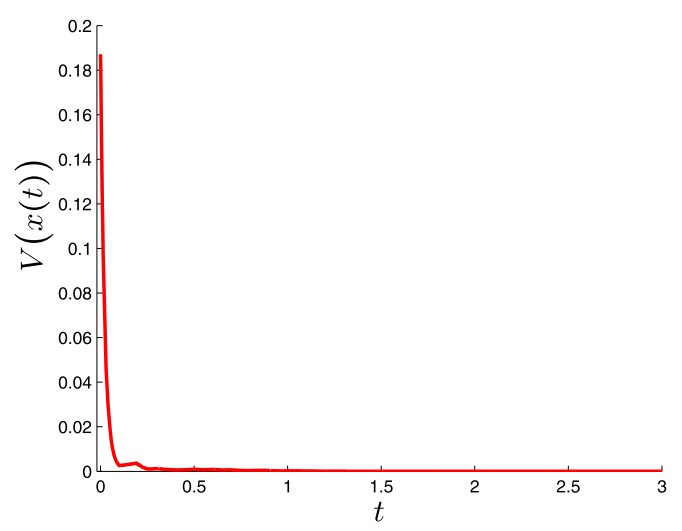

Fig. 6. Function $V$ along the switched system solution with initial condition $x_{0}=\left[\begin{array}{ll}3.5 & 0.8\end{array}\right]$.

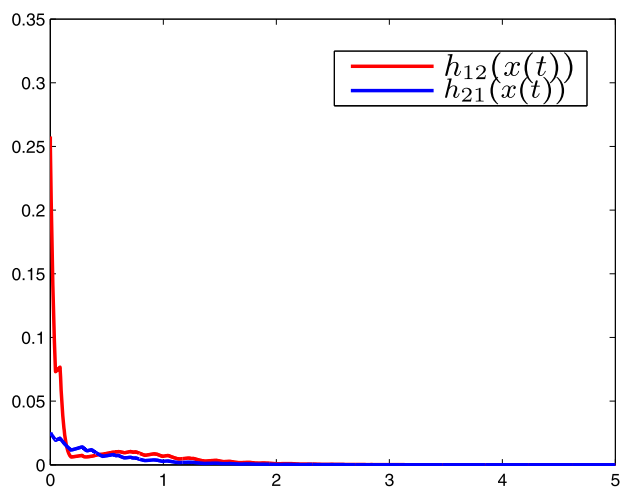

Fig. 7. Function $h_{12}(x(t))$ and $h_{21}(x(t))$ for initial condition $x_{0}=\left[\begin{array}{ll}3.5 & 0.8\end{array}\right]$.

\section{Conclusion}

The LMI conditions derived in this paper ensure the existence of a switching law that guarantees practical stability of nonlinear switched systems on the sense that trajectories are ultimately bounded and consequently converge to a bounded attractor. Estimates of the attractor and of the domain of attraction are obtained with the results of this paper in the form of invariant level sets of a Lyapunov-like function. Indeed, the LMIs formulation offers a systematic procedure to find auxiliary Lyapunov-like functions for switched nonlinear systems. The LMI conditions were derived using TS fuzzy description of each subsystems of the nonlinear switched system. Exploring an auxiliary system, which is a convex combination of the subsystems, it was proven that the switching law can be designed to ensure practical stability even when some of the subsystems are not practical stable, that is, have no ultimately bounded solutions. In comparison with other results in the literature, the proposed approach succeeded in maximizing the bounded attraction domain. The limitation of Theorem 1 is that we assume that set $\mathcal{D}$ is bounded. Further works in progress include checking if the set $\mathcal{D}$ is bounded into the LMIs constraints.

\section{Acknowledgement}

The authors would like to thank the Brazilian National Research Council (CNPq) for its support under grants 142246/2010-7, 306477/2013-0, 305486/2013-6 and 448391/2014-7. The authors also thank the reviewers for their valuable comments and suggestions. 


\section{References}

[1] N.S.D. Arrifano, V.A. Oliveira, R.A. Ramos, N.G. Bretas, R.V. Oliveira, Fuzzy stabilization of power systems in a co-generation scheme subject to random abrupt variations of operating conditions, IEEE Trans. Control Syst. Technol. 15 (2) (2007) 384-393.

[2] M. Rinehart, M. Dahleh, D. Reed, I. Kolmanovsky, Suboptimal control of switched systems with an application to the DISC engine, IEEE Trans. Control Syst. Technol. 16 (2) (2008) 189-201.

[3] D. Xie, Y. Wu, Stabilisation of time-delay switched systems with constrained switching signals and its applications in networked control systems, Control Theory Appl. 4 (10) (2010) 2120-2128.

[4] G.S. Deaecto, J.C. Geromel, F.S. Garcia, J.A. Pomilio, Switched affine systems control design with application to DC-DC converters, IET Control Theory Appl. 4 (7) (2010) 1201-1210.

[5] M.S. Branicky, Multiple Lyapunov functions and other analysis tools for switched and hybrid systems, IEEE Trans. Autom. Control 43 (4) (1998) 475-482.

[6] D. Liberzon, Switching in Systems and Control, Birkhäuser, Boston, USA, 2003.

[7] P. Colaneri, J.C. Geromel, A. Astolfi, Stabilization of continuous-time switched nonlinear systems, Syst. Control Lett. 57 (1) (2008) $95-103$.

[8] K. Tanaka, H.O. Wang, Fuzzy Control Systems Design and Analysis: A Linear Matrix Inequality Approach, John Wiley and Sons, New York, NY, 2001.

[9] L.A. Mozelli, R.M. Palhares, G.S.C. Avellar, A systematic approach to improve multiple Lyapunov function stability and stabilization conditions for fuzzy systems, Inf. Sci. 179 (8) (2009) 1149-1162.

[10] G. Feng, A survey on analysis and design of model-based fuzzy control systems, IEEE Trans. Fuzzy Syst. 14 (5) (2006) 676-697.

[11] Z. Wang, H. Wu, Fuzzy impulsive control for uncertain nonlinear systems with guaranteed cost, Fuzzy Sets Syst. 302 (1) (2016) $143-162$.

[12] Z. Zhang, C. Lin, B. Chen, New stability and stabilization conditions for T-S fuzzy systems with time delay, Fuzzy Sets Syst. 263 (2015) $82-91$.

[13] X. Xie, Z. Liu, X. Zhu, An efficient approach for reducing the conservatism of LMI-based stability conditions for continuous-time T-S fuzzy systems, Fuzzy Sets Syst. 263 (2015) 71-81.

[14] F.A. Faria, G.N. Silva, V.A. Oliveira, Reducing the conservatism of LMI-based stabilization conditions for TS fuzzy systems using fuzzy Lyapunov functions, Int. J. Syst. Sci. 44 (10) (2013) 1956-1969.

[15] K. Tanaka, T. Hori, H.O. Wang, A multiple Lyapunov function approach to stabilization of fuzzy control systems, IEEE Trans. Fuzzy Syst. 11 (4) (2003) 582-589.

[16] M. Bernal, T.M. Guerra, Generalized nonquadratic stability of continuous-time Takagi-Sugeno models, IEEE Trans. Fuzzy Syst. 18 (4) (2010) 815-822.

[17] T.M. Guerra, M. Bernal, Strategies to exploit non-quadratic local stability analysis, Int. J. Fuzzy Syst. 14 (3) (2012) $372-379$.

[18] T. González, M. Bernal, A. Sala, B. Aguiar, Cancellation-based nonquadratic controller design for nonlinear systems via Takagi-Sugeno models, IEEE Trans. Cybern. 47 (9) (2017) 2628-2638.

[19] D. Jabri, K. Guelton, N. Manamanni, A. Jaadari, C.D. Chinh, Robust stabilization of nonlinear systems based on a switched fuzzy control law, J. Control Eng. Appl. Inform. 14 (2) (2012) 40-49.

[20] W.A. Souza, M.C.M. Teixeira, R. Cardim, E. Assunção, On switched regulator design of uncertain nonlinear systems using Takagi-Sugeno fuzzy models, IEEE Trans. Fuzzy Syst. 22 (06) (2014) 1720-1727.

[21] Q. Zheng, H. Zhang, Asynchronous $H_{\infty}$ fuzzy control for a class of switched nonlinear systems via switching fuzzy Lyapunov function approach, Neurocomputing 182 (2016) 178-186.

[22] A. Jaballi, A.E. Hajjaji, A. Sakly, Reducing conservativeness of stabilization conditions for switched TS fuzzy systems, Neurocomputing 193 (2016) 51-57.

[23] F.A. Faria, M.C. Valentino, V.A. Oliveira, A fuzzy Lyapunov function approach for stabilization and $\mathcal{H}_{\infty}$ control of switched T-S fuzzy systems, Appl. Math. Model. 38 (2014) 4817-4834.

[24] H. Lin, P.J. Antsaklis, Stability and stabilizability of switched linear systems: a survey of recent results, IEEE Trans. Autom. Control 54 (2) (2009) 308-322.

[25] A. Bacciotti, L. Mazzi, From Artstein-Sontag theorem to the min-projection strategy, Trans. Inst. Meas. Control 32 (6) (2010) 571-581.

[26] X. Xu, G. Zhai, Some results on practical asymptotic stabilizability of switched systems, in: 44th IEEE Conference on Decision and Control, and the European Control Conference, Seville, Spain, 2005, pp. 3998-4003.

[27] J.F. Sturm, Using SeDuMi 1.02, a matlab toolbox for optimization over symmetric cones, Optim. Methods Softw. 11-12 (1999) 625-653, http://citeseer.ist.psu.edu/sturm99using.html.

[28] T. Taniguchi, K. Tanaka, H. Ohtake, H.O. Wang, Model construction, rule reduction, and robust compensation for generalized form of TakagiSugeno fuzzy systems, IEEE Trans. Fuzzy Syst. 9 (4) (2001) 525-537.

[29] J.C. Geromel, M.C. de Oliveira, L. Hsu, Lmi characterization of structural and robust stability, Linear Algebra Appl. 285 (1) (1998) 69-80, https://doi.org/10.1016/S0024-3795(98)10123-4, http://www.sciencedirect.com/science/article/pii/S0024379598101234.

[30] D.H. Lee, J.B. Park, Y.H. Joo, A fuzzy Lyapunov function approach to estimating the domain of attraction for continuous-time Takagi-Sugeno fuzzy systems, Inf. Sci. 185 (1) (2012) 230-248.

[31] J. Löfberg, Yalmip: a toolbox for modeling and optimization in MATLAB, in: Proceedings of the CACSD Conference, Taipei, Taiwan, 2004, pp. 284-289, http://control.ee.ethz.ch/ joloef/yalmip.php. 